\title{
A Geometric Proof of Total Positivity for Spline Interpolation
}

\author{
By C. de Boor* and R. DeVore*,**
}

\begin{abstract}
Simple geometric proofs are given for the total positivity of the B-spline collocation matrix and the variation diminishing property of the B-spline representation of a spline.
\end{abstract}

1. Introduction. Perhaps a better title would be "Adding a knot can be illuminating" since the purpose of this note is to show how this idea can be used to give simple proofs of several important properties of B-splines, including the total positivity of the B-spline collocation matrix, and the sign variation diminishing property of the B-spline representation. We show (as did Lane and Riesenfeld [6]) that variation diminution follows immediately from the fact that a B-spline on a given grid is a nonnegative linear combination of B-splines on a refined grid. We use the same fact to prove the nonnegativity of any minor of the collocation matrix and, with a bit more care, even characterize which of these minors are positive.

The total positivity of the collocation matrix was originally proved by S. Karlin [5] in his development of the general theory of total positivity. He derives variation diminution from total positivity, as can be done for arbitrary matrices. In fact, it is well-known that variation diminution is equivalent to sign regularity. But, because of the great practical interest in these spline properties, it is desirable to have a more direct, spline-specific proof. Such a proof was given in [3], but again variation diminution was derived from total positivity. We obtain both properties directly. This was motivated in part by the work of J. Lane and R. Riesenfeld [6], who gave a direct proof of variation diminution based on spline evaluation algorithms used in computer-aided design which can be interpreted as "adding knots". But it seems to be much simpler to follow Böhm's idea [1] of adding one knot at a time as we do in Section 3. We note that Jia [4] has done related work concerning the total positivity of the discrete B-spline collocation matrix.

Let $k>0$ be a fixed integer which is the order of the splines. We call $\mathbf{t}:=\left(t_{i}\right)_{1}^{n+k}$ a knot sequence if $t_{i} \leqslant t_{i+1}, 1 \leqslant i<n+k$ and $t_{i}<t_{i+k}, i=1, \ldots, n$. The B-splines of order $k$ for this knot sequence are given by

$$
N_{i}(x):=N_{i, \mathrm{t}}(x):=\left(t_{i+k}-t_{i}\right)\left[t_{i}, \ldots, t_{i+k}\right](\cdot-x)_{+}^{k-1}, \quad i=1, \ldots, n,
$$

Received March 19, 1984; revised February 8, 1985.

1980 Mathematics Subject Classification. Primary 41A15, 41A25, 41 A50.

Key words and phrases. Spline interpolation, total positivity, variation diminishing.

* Sponsored by the United States Army under Contract No. DAAG29-80-C-0041.

** Supported by the National Science Foundation under Grant. No. DMS 8320562.

1985 American Mathematical Society $0025-5718 / 85 \$ 1.00+\$ .25$ per page 
where $\left[t_{i}, \ldots, t_{i+k}\right]$ denotes a $k$ th order divided difference and $u_{+}:=\max \{u, 0\}$. It follows that $N_{i} \geqslant 0$ and $\operatorname{supp} N_{i}=\left(t_{i}, t_{i+k}\right)$. On each interval $\left(t_{j}, t_{j+1}\right), N_{i}$ is a polynomial of order $k$ (degree $<k$ ). The B-splines are linearly independent and $\sum N_{j} \equiv 1$ on $\left[t_{k}, t_{n}\right]$. In particular, if the number $x \in\left(t_{1}, t_{n+k}\right)$ appears exactly $k-1$ times in $\mathbf{t}$, then there is only one B-spline which is nonzero at $x$ and its value at $x$ is one. For these and other properties of B-splines, see [3].

2. Knot Refinement. We say that the knot sequence $s$ is a refinement of $\mathbf{t}$ if $\mathbf{s}$ contains $\mathbf{t}$ as a subsequence. Our only tool in the subsequent arguments is the observation that

any $B$-spline $N_{j}=N_{j, \mathrm{t}}$ is a positive linear combination of some of

the $B$-splines $N_{j}^{\prime}:=N_{j, s}$ for the refined knot sequence $\mathbf{s}$.

Precisely,

$$
N_{j}=\sum \alpha_{j}(i) N_{i}^{\prime}
$$

with $\alpha_{j}$ nonnegative, and $\operatorname{supp} \alpha_{j}=[l, u]$, where $\left(s_{l}, s_{u+k}\right)$ is the smallest segment of $\mathbf{s}$ containing $\left(t_{j}, \ldots, t_{j+k}\right)$ as a subsequence.

We first prove (2.1) for the special case that

$$
\mathbf{s}=\left(\ldots, t_{\nu-1}, s_{\nu}, t_{\nu}, \ldots\right),
$$

i.e., $\mathbf{S}$ is obtained from $\mathbf{t}$ by the addition of the knot $s_{\nu}$ (satisfying $t_{\nu-1} \leqslant s_{\nu} \leqslant t_{\nu}$, of course). Then

$$
N_{j}= \begin{cases}N_{j}^{\prime} & \text { for } j+k<\nu, \\ N_{j+1}^{\prime} & \text { for } \nu \leqslant j\end{cases}
$$

For $j<\nu \leqslant j+k$, we have two ways of writing the divided difference $\left[s_{j}, \ldots, s_{j+k+1}\right]$ :

$$
\frac{S_{j+1}-S_{j}}{s_{j+k+1}-s_{j}}=\left[s_{j}, \ldots, s_{j+k+1}\right]=\frac{T_{j}-S_{j}}{s_{j+k+1}-s_{\nu}},
$$

with $S_{i}:=\left[s_{i}, \ldots, s_{i+k}\right], T_{i}:=\left[t_{i}, \ldots, t_{i+k}\right]$. Therefore, since $s_{j+k+1}=t_{j+k}$ and $s_{j}=t_{j}$,

$$
\left(t_{j+k}-t_{j}\right) T_{j}=\left(s_{\nu}-s_{j}\right) S_{j}+\left(s_{j+k+1}-s_{\nu}\right) S_{j+1} \text {, }
$$

hence

$$
N_{j}=\frac{s_{\nu}-s_{j}}{s_{j+k}-s_{j}} N_{j}^{\prime}+\frac{s_{j+k+1}-s_{\nu}}{s_{j+k+1}-s_{j+1}} N_{j+1}^{\prime}, \quad j<\nu \leqslant j+k .
$$

We can combine this with (2.2) into one formula, as follows:

$$
N_{j}=\left(1-\gamma_{j}\right) N_{j}^{\prime}+\gamma_{j+1} N_{j+1}^{\prime}, \quad \text { all } j,
$$

with

$$
\gamma_{j}:=\min \left\{\frac{\left(s_{j+k}-s_{\nu}\right)_{+}}{s_{j+k}-s_{j}}, 1\right\}, \quad \text { all } j
$$

Consequently,

$$
\operatorname{supp} \alpha_{j}= \begin{cases}{[j],} & \text { if } t_{j+k} \leqslant s_{\nu}, \\ {[j, j+1],} & \text { if } t_{j}<s_{\nu}<t_{j+k}, \\ {[j+1],} & \text { if } s_{\nu} \leqslant t_{j}\end{cases}
$$

and this finishes the proof of (2.1) for this case. 
The general case follows from the repeated application of this special case, by induction: Suppose that $\mathbf{r}$ is, in turn, a refinement of $\mathbf{s}$, hence

$$
N_{i}^{\prime}=\sum \alpha_{i}^{\prime}(l) N_{l}^{\prime \prime}
$$

with $N_{l}^{\prime \prime}:=N_{l, \mathbf{r}}$. Then, it follows that

$$
N_{j}=\sum \beta_{j}(l) N_{l}^{\prime \prime}, \quad \text { with } \beta_{j}(l)=\sum_{i} \alpha_{j}(i) \alpha_{i}^{\prime}(l) .
$$

Therefore, $\beta_{j} \geqslant 0$, since we already know that $\alpha_{j}, \alpha_{j}^{\prime} \geqslant 0$. Further,

$$
\operatorname{supp} \beta_{j}=\bigcup_{i \in \operatorname{supp} \alpha_{j}} \operatorname{supp} \alpha_{i}^{\prime}=\left[l^{\prime}, u^{\prime}\right]
$$

with $\left(r_{l^{\prime}}, \ldots, r_{u^{\prime}+k}\right)$ the smallest segment of $\mathbf{r}$ containing $\left(s_{l}, \ldots, s_{u+k}\right)$ as a subsequence. But, since $[l, u]$ is the support of $\alpha_{j}$, i.e., $\left(s_{l}, \ldots, s_{u+k}\right)$ is the smallest segment of $\mathbf{s}$ containing $\left(t_{j}, \ldots, t_{j+k}\right)$, it follows that $\left(r_{l^{\prime}}, \ldots, r_{u^{\prime}+k}\right)$ is also the smallest segment of $\mathbf{r}$ containing $\left(t_{j}, \ldots, t_{j+k}\right)$.

The coefficient function $\alpha_{j}$ in (2.1) has been called a discrete B-spline. The above argument shows that the matrix $\left(\alpha_{j}(i)\right)$ is the product of bidiagonal matrices with nonnegative entries, hence totally positive by Cauchy-Binet. This is the basic idea behind the proof of such total positivity in Jia [4].

3. Variation Diminution. We use the customary notation $S^{-}(\alpha)$ for the number of (strong) sign changes in the sequence or function $\alpha$. We want to show that $S^{-}\left(\sum \lambda_{j} N_{j}\right) \leqslant S^{-}(\lambda)$, i.e., the spline $f:=\sum_{1}^{n} \lambda_{j} N_{j}$ changes sign no more often than its coefficient sequence $\lambda$. This follows from:

(i) if $f:=\sum \lambda_{j} N_{j}=\sum \lambda_{j}^{\prime} N_{j}^{\prime}$ with $N_{j}^{\prime}:=N_{j, \mathbf{s}}$ and $\mathbf{s}$ a refinement of $\mathbf{t}$, then $S^{-}(\lambda) \geqslant S^{-}\left(\lambda^{\prime}\right)$;

(ii) if, in addition, $x \in\left(t_{1}, t_{n+k}\right)$ appears as a knot in $\mathbf{s}$ with (exact) multiplicity $k-1$, then $\lambda_{j}^{\prime}=f(x)$ for some $j$.

Property (ii) is clear. To prove property (i), we first consider the special case when $\mathbf{s}$ is obtained from $\mathbf{t}$ by the addition of a single knot. In that case, we infer from (2.4) that

$$
\sum_{1}^{n} \lambda_{j} N_{j}=\sum_{1}^{n} \lambda_{j}\left(\left(1-\gamma_{j}\right) N_{j}^{\prime}+\gamma_{j+1} N_{j+1}^{\prime}\right)
$$

Therefore,

$$
\sum \lambda_{j} N_{j}=\sum \lambda_{j}^{\prime} N_{j}^{\prime} \quad \text { with } \lambda_{j}^{\prime}:=\gamma_{j} \lambda_{j-1}+\left(1-\gamma_{j}\right) \lambda_{j}, \text { all } j .
$$

(Here, we set $\lambda_{0}:=0$.) Since $\gamma_{j} \in[0,1]$, this implies that $S^{-}\left(\lambda_{j-1}, \lambda_{j}^{\prime}, \lambda_{j}\right)=$ $S^{-}\left(\lambda_{j-1}, \lambda_{j}\right)$. Therefore, $S^{-}(\lambda)=S^{-}\left(\ldots, \lambda_{j-1}, \lambda_{j}^{\prime}, \lambda_{j}, \lambda_{j+1}^{\prime}, \ldots\right) \geqslant S^{-}\left(\lambda^{\prime}\right)$. This shows (3.1(i)) for a single knot refinement. But then by induction we get (3.1(i)) for any refinement.

Theorem 1. (Variation Diminishing Property.) $S^{-}\left(\sum \lambda_{j} N_{j}\right) \leqslant S^{-}(\lambda)$.

Proof. Let $f=\sum_{1}^{n} \lambda_{j} N_{j}$. We want to show that, for any increasing real sequence $\left(z_{i}\right)_{1}^{l}, S^{-}\left(\left(f\left(z_{i}\right)\right)\right) \leqslant S^{-}(\lambda)$. We can assume that the $z_{i}$ are not knots and that $z_{i} \in\left(t_{1}, t_{n+k}\right)$ (since $f \equiv 0$ outside this interval). Let $\mathbf{s}$ be a knot refinement of $\mathbf{t}$ such that each $z_{i}$ appears exactly $k-1$ times in $\mathbf{s}$. Then, from (3.1(ii)), the sequence $\left(f\left(z_{i}\right)\right)_{1}^{l}$ is a subsequence of $\lambda$ and the desired result follows from (3.1(i)). 
It is sometimes useful to visualize the coefficients $\left(\lambda_{j}\right)$ geometrically. If $t_{j}^{*}:=$ $\left(t_{j+1}+\cdots+t_{j+k-1}\right) /(k-1)$, then the continuous piecewise-linear function $P(f, \mathbf{t})$ with vertices $\left(t_{j}^{*}, \lambda_{j}\right), j=1, \ldots, n$, is called the B-polygon of $f$. This polygon changes sign exactly as often as $\lambda$. For a single knot refinement $\mathbf{s}$ of $\mathbf{t}$, the points $s_{j}^{*}$ are related to $t_{j}^{*}$ as in (3.2), i.e.,

$$
s_{j}^{*}=\gamma_{j} t_{j-1}^{*}+\left(1-\gamma_{j}\right) t_{j}^{*} .
$$

Hence, the vertices of $P(f, \mathbf{s})$ lie on $P(f, \mathbf{t})$; which is another way of viewing property (3.1(i)).

4. Spline Interpolation. We now consider spline interpolation at nodes $\left(x_{i}\right)_{1}^{n}$, $x_{1}<x_{2}<\cdots<x_{n}$ (later we allow coalescence). Given $\left(y_{i}\right)_{1}^{n}$, we have the interpolation problem

$$
\sum_{j=1}^{n} \lambda_{j} N_{j}\left(x_{i}\right)=y_{i}, \quad i=1, \ldots, n,
$$

with coefficient matrix

$$
A:=A_{\mathrm{t}}:=\left(N_{j}\left(x_{i}\right)\right)_{i, j=1}^{n} .
$$

In case $x_{i}=t_{j}$, we require that this point appear at most a total of $k$ times in $\mathbf{x}$ and t.

We will show that $A$ is totally positive and furthermore characterize which minors of $A$ are strictly positive. For this, let $B$ be a square submatrix of $A$,

$$
B=A(I, J):=\left(N_{j}\left(x_{i}\right)\right)_{i \in I, j \in J},
$$

with $I$ and $J$ subsequences of $(1,2, \ldots, n)$ of the same length,

$$
I=:\left(i_{1}, \ldots, i_{m}\right), \quad J=:\left(j_{1}, \ldots, j_{m}\right),
$$

say. We call such a submatrix "good" if all its diagonal entries are nonzero. This is a natural distinction to make here because

$$
\text { if } B \text { is not "good", then } \operatorname{det} B=0 \text {. }
$$

Indeed, assume that $N_{j_{p}}\left(x_{i_{p}}\right)=0$ for some $p$. Then $x_{i_{p}} \notin\left(t_{j_{p}}, t_{j_{p}+k}\right)$. Assume that $x_{i_{p}} \leqslant t_{j_{p}}$. Then, $N_{j}\left(x_{q}\right)=0$ for $q \leqslant i_{p}, j \geqslant j_{p}$, and this shows that columns $p, \ldots, m$ of $B$ have nonzero entries only in rows $p+1, \ldots, m$, hence are linearly dependent. So, det $B=0$. The argument for the case $x_{i_{p}} \geqslant t_{j_{p}+k}$ is similar.

Next, we write det $B$ as a linear combination of determinants of the form $A^{\prime}(I, K)$ with

$$
A^{\prime}:=\left(N_{j}^{\prime}\left(x_{i}\right)\right)
$$

and $\left(N_{j}^{\prime}\right)$ the B-splines for a refinement $\mathbf{s}$ of $\mathbf{t}$. Precisely, we claim that, for a certain nonnegative $a_{J}$,

$$
\operatorname{det} A(I, J)=\sum^{+} a_{J}(K) \operatorname{det} A^{\prime}(I, K)
$$

with the superscript " +" indicating that the sum is only over increasing K. Further,

$$
\operatorname{supp} a_{J}=\operatorname{supp} \alpha_{J}
$$

where $\alpha_{J}(K):=\alpha_{j_{1}}\left(k_{1}\right) \cdots \alpha_{j_{m}}\left(k_{m}\right)$ and the $\alpha_{j}$ are as in (2.1). 
For the proof, we consider first the special case that $\mathbf{s}$ is obtained from $\mathbf{t}$ by the addition of a single knot. Since $N_{j}=\sum \alpha_{j}(i) N_{i}^{\prime}$ by (2.1), the linearity of the determinant as a function of the columns gives

$$
\operatorname{det} A(I, J)=\sum \alpha_{J}(K) \operatorname{det} A^{\prime}(I, K)
$$

with $\alpha_{J}(K):=\alpha_{j_{1}}\left(k_{1}\right) \cdots \alpha_{j_{m}}\left(k_{m}\right)$. Recall from (2.5) that $\operatorname{supp} \alpha_{j} \subseteq[j, j+1]$. Therefore, retaining in (4.5) only terms with $\alpha_{J}(K) \neq 0$, we have $k_{p}=j_{p}$ or $j_{p}+1$, all $p$. Thus $K$ is strictly increasing unless $k_{p}=k_{p+1}$ for some $p$ (possible in case $\left.j_{p}+1=j_{p+1}\right)$. But, in the latter case, the determinant is trivially zero and hence can be ignored. This finishes the proof of (4.4) for this special case.

We prove the general case by induction on the length difference $d:=|\mathbf{s}|-|\mathbf{t}|$, having just proved it for $d=1$. Assuming it correct for a given $d$, let $\mathbf{r}$ be a refinement of $\mathbf{t}$ with $|\mathbf{r}|-|\mathbf{t}|=d+1$ and let $\mathbf{s}$ be a one-point refinement of $\mathbf{t}$ which is refined by $\mathbf{r}$. Then, with

$$
A^{\prime \prime}:=\left(N_{j}^{\prime \prime}\left(x_{i}\right)\right) \text { and } N_{j}^{\prime \prime}:=N_{j, \mathrm{r}}, \text { all } j,
$$

we have $N_{j}^{\prime}=\sum \alpha_{j}^{\prime}(l) N_{l}^{\prime \prime}$. Further, from (4.5) and the induction hypothesis,

$$
\operatorname{det} A(I, J)=\sum^{+} b_{J}(L) \operatorname{det} A^{\prime \prime}(I, L)
$$

with

$$
b_{J}(L):=\sum^{+} \alpha_{J}(K) a_{K}^{\prime}(L) \geqslant 0,
$$

which makes $(4.4 \mathrm{a})$ obvious.

The proof of (4.4b) is a bit more complicated. It can be skipped if only the total positivity of $A$ is of interest. We must show that $\operatorname{supp} b_{J}=\operatorname{supp} \beta_{J}$, with $\beta_{J}(L):=\beta_{j_{1}}\left(l_{1}\right) \cdots \beta_{j_{m}}\left(l_{m}\right)$. Suppose first that $\beta_{J}(L)=0$. Then $\beta_{j}(l)=0$ for some $j \in J, l \in L$. Therefore, from (2.6), $\sum \alpha_{j}(i) \alpha_{i}^{\prime}(l)=0$, and, since all terms in this sum are nonnegative, they must all be zero. Thus, $\alpha_{J}(K) \alpha_{K}^{\prime}(L)=0$ for all $K$. But by induction hypothesis, supp $a_{K}^{\prime}=\operatorname{supp} \alpha_{K}^{\prime}$, therefore also $\alpha_{J}(K) a_{K}^{\prime}(L)=0$ for all $K$. We conclude with (4.6) that $\operatorname{supp} b_{J} \subset \operatorname{supp} \beta_{J}$.

To see that $\operatorname{supp} b_{J} \supset \operatorname{supp} \beta_{J}$, we must show that

$$
\beta_{J}(L) \neq 0 \text { implies } \alpha_{J}(K) \alpha_{K}^{\prime}(L) \neq 0 \text { for some increasing } K \text {. }
$$

Since supp $a_{K}^{\prime}=\operatorname{supp} \alpha_{K}$, this implies that $\alpha(K) a_{K}^{\prime}(L) \neq 0$ for this increasing $K$, hence also $b_{J}(L) \neq 0$ from (4.6).

For the proof of (4.7), it is sufficient to show the existence of a $K$ with

$$
k_{p} \in A_{j_{p}}:=\left\{i: \alpha_{j_{p}}(i) \alpha_{i}^{\prime}\left(l_{p}\right) \neq 0\right\}, \quad \text { all } p,
$$

and $k_{p}<k_{p+1}$, all $p$. Then $A_{j} \subseteq\{j, j+1\}$ and, since

$$
\beta_{j}(l)=\alpha_{j}(j) \alpha_{j}^{\prime}(l)+\alpha_{j}(j+1) \alpha_{j+1}^{\prime}(l),
$$

$\beta_{J}(L) \neq 0$ implies that

$$
\varnothing \neq A_{j}, \quad \text { all } j \in J .
$$

Hence, the existence of $K$ satisfying (4.8) is assured. To finish the proof, we must show that it is possible to choose such a $K$ which is also increasing. If $A_{j_{p}} \cap A_{j_{p+1}}=$ $\varnothing$, then we have $k_{p}<k_{p+1}$ for any $K$ satisfying (4.8). Thus, we only have to 
consider how to choose the components of $K$ corresponding to a connected component $A_{j_{p}}, \ldots, A_{j_{q}}$. By this we mean that

$$
A_{j_{\nu}} \cap A_{j_{\nu+1}} \neq \varnothing \quad \text { for } p \leqslant \nu<q
$$

while, for any $i \neq j_{p}, \ldots, j_{q}$,

$$
A_{i} \cap A_{j_{\nu}}=\varnothing \text { for } p \leqslant \nu \leqslant q .
$$

Then we can write $\left(j_{p}, \ldots, j_{q}\right)=\left(j, j+1, \ldots, j^{\prime}\right)$, hence, $q-p=j^{\prime}-j$. Further, $i \in A_{i}$ for $i=j+1, \ldots, j^{\prime}$. Hence, if also $j \in A_{j}$, then the choice $k_{\nu}=j_{\nu}$, all $\nu$, will do. In the same way, we have $i+1 \in A_{i}$ for $i=j, \ldots, j^{\prime}-1$. Hence, if $j^{\prime}+1 \in A_{j^{\prime}}$, then the choice $k_{\nu}=j_{\nu}+1$, all $\nu$, will do. We claim that the remaining case

$$
j \notin A_{j} \quad \text { and } \quad j^{\prime}+1 \notin A_{j^{\prime}}
$$

cannot occur since it would imply that there are at least $k$ entries in $\mathbf{r}$ between $r_{l_{p}}$ and $r_{l_{p}+k}$. Indeed, with supp $\alpha_{j}^{\prime}=:[l, u]$, it would follow that $u<l_{p}$, while also $l_{q}<l^{\prime}$, with supp $\alpha_{j^{\prime}+1}^{\prime}=:\left[l^{\prime}, u^{\prime}\right]$. Further, let $s_{\nu}$ be the additional knot in $\mathbf{s}$. Then, by (2.5), $A_{i} \cap A_{i+1} \neq \varnothing$ implies $s_{i+1}<s_{\nu}<s_{i+k+1}, i=j, \ldots, j^{\prime}$, therefore $s_{j^{\prime}+1}<s_{j+k}$, and so $l^{\prime}<u+k$ while also $p+k-q-1=j+k-\left(j^{\prime}+1\right) \leqslant u+k-l^{\prime}$. This would imply that

$$
l_{p}<l_{p+1}<\cdots<l_{q}<l^{\prime}<u+k<l_{p}+k
$$

hence $k=l_{p}+k-l_{p} \geqslant 1+\left(u+k-l^{\prime}\right)+1+q-p \geqslant 1+(p+k-q-1)+$ $1+q-p=k+1$.

THEOREM 2. The matrix $A$ of (4.2) is totally positive. Moreover, the submatrix $B$ of $A$ formed by rows $i_{1}, \ldots, i_{m}$ and columns $j_{1}, \ldots, j_{m}$ has a positive determinant if and only if it is "good", i.e.,

$$
x_{i_{\nu}} \in \operatorname{supp} N_{j_{\nu}}, \quad \nu=1, \ldots, r .
$$

Proof. We already proved that $\operatorname{det} B=0$ unless $B$ is "good". Now, to prove that a "good" $B$ has a positive determinant, we choose a refinement $\mathbf{s}$ of $\mathbf{t}$ so fine that

$$
\text { for each } i \in I, N_{j}^{\prime}\left(x_{i}\right) \neq 0 \text { implies that } N_{j}^{\prime}\left(x_{p}\right)=0 \text { for all } p \neq i \text {. }
$$

Then each $A^{\prime}(I, K)$ appearing in (4.4a) has at most one nonzero entry in each column, hence is "good", therefore, nonzero, only if it is diagonal, in which case its determinant is obviously positive. To finish the proof, we must show that at least one of the matrices appearing in the sum in (4.4a) with a positive coefficient is "good". Here is one such. Choose $K$ so that $s_{k_{p}}$ is the first point in $\mathbf{s}$ to the left of $x_{i_{p}}$, $p=1, \ldots, m$. Since $N_{j_{p}}\left(x_{i_{p}}\right) \neq 0$, this implies that $\alpha_{j_{p}}\left(k_{p}\right) \neq 0$, all $p$.

Corollary (I. Schoenberg AND A. Whitney [7]). The interpolation problem (4.1) has a unique solution for all $\left(y_{i}\right)_{1}^{n}$ if and only if $x_{i} \in \operatorname{supp} N_{i}, i=1, \ldots, n$.

We can also allow coalescence of the interpolation nodes. If $\left(z_{i}\right)$ is such a nondecreasing sequence of nodes, then we can think of it as the limit of strictly increasing sequences $\left(x_{i}\right)$. Correspondingly, repetition of a $z_{i}$ corresponds to repeated or osculatory interpolation, i.e., the matching of higher derivatives. Precisely, 
(4.1) becomes

$$
\sum_{1}^{n} \lambda_{j} D^{\mu_{i}} N_{j}\left(z_{i}\right)=y_{i}, \quad i=1, \ldots, n,
$$

where $\mu_{i}$ is the number of $j<i$ for which $z_{j}=z_{i}$. We still require that any point appear at most $k$ times totally in $\mathbf{z}$ and $\mathbf{t}$. The coefficient matrix of $(4.10)$ is

$$
A:=A_{\mathrm{t}, \mathrm{z}}:=\left(D^{\mu_{i}} N_{j}\left(z_{i}\right)\right)_{i, j=1}^{n} .
$$

It is clear that $A$ need not be totally positive since entries involving derivatives may be negative. However, as a well-known argument shows, if $M$ is a minor formed by rows $i_{1}, \ldots, i_{m}$ and columns $j_{1}, \ldots, j_{m}$ with the property

$$
i_{\nu-1}<i_{\nu}-1 \text { implies } z_{i_{\nu}-1}<z_{i_{\nu}}, \quad \nu=1, \ldots, m,
$$

then $M \geqslant 0$. In fact, if $M(\mathbf{x})$ denotes a minor corresponding to distinct nodes $\mathbf{x}=\left(x_{1}, \ldots, x_{m}\right)$, then subtracting row one from row two shows that $M(\mathbf{x}) /\left(x_{2}-x_{1}\right)$ converges as $x_{2} \rightarrow x_{1}$ to the minor $M^{\prime}$ which replaces row two of $M(\mathbf{x})$ by first derivatives at $x_{1}$. Hence, $M^{\prime} \geqslant 0$. Using this type of limiting process, we see that any minor $M$ satisfying (4.12) is nonnegative.

We can also characterize those $M$ satisfying (4.12) which are positive, namely, they satisfy

$$
z_{i_{\nu}} \in \operatorname{supp} N_{j_{\nu}}, \quad \nu=1, \ldots, m .
$$

The necessity of (4.13) is proved in the same way that the necessity of (4.9) was established.

The sufficiency of (4.13) is proved by making slight modifications to the earlier proof. For this, it will be convenient to allow a point $z_{i}$ to appear a total of more than $k$ times in $\mathbf{s}$ and $\mathbf{x}$. This is acceptable provided we stipulate that all B-splines and their derivatives be interpreted as right limits at such $z_{i}$, that is at $z_{i}^{+}$. With this, let $\mathbf{s}$ be a refinement of $\mathbf{t}$ such that each node $z_{i}$ appears as a knot in $\mathbf{s}$ exactly $k$ times, and similarly each $t_{i}$ appears in $\mathbf{s}$ exactly $k$ times. If $J$ satisfies (4.13), we choose $L$ so that $s_{l_{p}}=z_{i_{p}}$ and the number of $j<l_{p}$ with $s_{j}=s_{l_{p}}$ is $\mu_{i_{p}}$. Since the coefficients $\alpha(K)$ in (4.4a) are independent of $\mathbf{x}$, we then obtain $\operatorname{det} A(I, J)$ as a positive combination of certain (nonnegative) minors of $A^{\prime}:=A_{\mathrm{s}, \mathbf{z}}$. In particular, the submatrix $A^{\prime}(J, L)$ will appear in that sum with positive coefficient since $\alpha_{J}(L)>0$, and det $A^{\prime}(J, L)>0$ since $A^{\prime}(J, L)$ is lower triangular with positive diagonal. We have therefore proved the following theorem.

THEOREM 3. For the matrix $A$ of (4.11), and each $I, J$ satisfying (4.12), $\operatorname{det} A(I, J)$ $\geqslant 0$. This minor is positive if and only if (4.13) is satisfied. In particular, (4.10) has $a$ unique solution if and only if $z_{i} \in \operatorname{supp} N_{i}, i=1, \ldots, n$.

Mathematics Research Center

610 Walnut Street

Madison WI 53705

Department of Mathematics and Statistics

University of South Carolina

Columbia, South Carolina 29208 
1. W. Вонм, “Inserting new knots into B-spline curves," Computer-Aided Design, v. 12, 1980, pp. 199-201.

2. C. DE BooR, "Total positivity of the spline collocation matrix," Indiana Univ. Math. J., v. 25, 1976, pp. 541-551.

3. C. DE Boor, A Practical Guide to Splines, Applied Mathematical Sciences, Vol. 27, Springer-Verlag, Berlin and New York, 1978.

4. JIA RONG-QING, "Total positivity of the discrete spline collocation matrix", J. Approx. Theory, v. 39. 1983, pp. 11-23.

5. S. Karlin, Total Positivity, Vol. I, Stanford Univ. Press, Stanford, Calif., 1968.

6. J. LANE \& R. RIESENFELD, “A geometric proof for the variation diminishing property of B-spline approximation," J. Approx. Theory, v. 37, 1983, pp. 1-4.

7. I. J. Schoenberg \& A. Whitney, "On Pólya frequency functions. III," Trans. Amer. Math. Soc., v. 74, 1953, pp. 246-259. 